Catherine M. Roe, PhD

Anne M. Fagan, $\mathrm{PhD}$

Elizabeth A. Grant, PhD

David M. Holtzman, MD

John C. Morris, MD

Correspondence to

Dr. Roe:

cathyr@wustl.edu
Supplemental data at www.neurology.org

\section{CSF biomarkers of Alzheimer disease}

\author{
"Noncognitive" outcomes
}

ABSTRACT

Objectives: To test whether CSF Alzheimer disease biomarkers ( $\beta$-amyloid 42 [A $\beta_{42}$ ], tau, phosphorylated tau at threonine $181\left[p^{2} u_{181}\right]$, tau/A $\beta_{42}$, and $\left.p \operatorname{tau}_{181} / A \beta_{42}\right)$ predict future decline in noncognitive outcomes among individuals cognitively normal at baseline.

Methods: Longitudinal data from participants $(N=430)$ who donated CSF within 1 year of a clinical assessment indicating normal cognition and were aged 50 years or older were analyzed. Mixed linear models were used to test whether baseline biomarker values predicted future decline in function (instrumental activities of daily living), weight, behavior, and mood. Clinical Dementia Rating Sum of Boxes and Mini-Mental State Examination scores were also examined.

Results: Abnormal levels of each biomarker were related to greater impairment with time in behavior $(p<0.035)$ and mood $(p<0.012)$ symptoms, and more difficulties with independent activities of daily living $(p<0.012)$. However, biomarker levels were unrelated to weight change with time $(p>0.115)$. As expected, abnormal biomarker values also predicted more rapidly changing MiniMental State Examination $(p<0.041)$ and Clinical Dementia Rating Sum of Boxes $(p<0.001)$ scores compared with normal values.

Conclusions: CSF biomarkers among cognitively normal individuals are associated with future decline in some, but not all, noncognitive Alzheimer disease symptoms studied. Additional work is needed to determine the extent to which these findings generalize to other samples. Neurology ${ }^{\circledast} 2013 ; 81: 2028-2031$

\section{GLOSSARY}

$\mathbf{A} \boldsymbol{\beta}_{\mathbf{4 2}}=\boldsymbol{\beta}$-amyloid 42; $\mathbf{A D}=$ Alzheimer disease $\mathbf{C D R}=$ Clinical Dementia Rating; $\mathbf{F A Q}=$ Functional Assessment Questionnaire; $\mathbf{G D S}=$ Geriatric Depression Scale; IADL = instrumental activities of daily living; $\mathbf{M C I}=$ mild cognitive impairment; MMSE = Mini-Mental State Examination; NPI-Q = Neuropsychiatric Inventory Questionnaire; $\mathbf{p t a u}_{181}=$ phosphorylated tau at threonine 181 .

Alzheimer disease $(\mathrm{AD})$ biomarkers can identify "preclinical AD," a state in which AD pathology is present in the brain but the individual has no dementia symptoms or detectable cognitive impairment. It is thought that these biomarkers may become abnormal many years before problems in memory and thinking appear. ${ }^{1-3}$ Biomarkers now are being used in "secondary prevention" trials to identify cognitively normal individuals who are likely to develop symptomatic AD in the future. When dementia symptoms are manifest, substantial neuronal death has already occurred. Therefore, it may be that waiting to administer drugs at the "late" symptomatic stage of $\mathrm{AD}$ is responsible for the failure of treatment trials with "disease-modifying" agents.

Earlier work has shown that CSF biomarkers of $\mathrm{AD}$ reflecting soluble $\beta$-amyloid $(\mathrm{A} \beta)$, the principal component of amyloid plaques, and tau, the principal component of tangles, predict incident cognitive impairment and dementia. However, "noncognitive" outcomes must also be carefully and systematically assessed in clinical trials of disease-modifying treatments for AD. ${ }^{4}$ These outcomes help to more fully reflect the impact of $\mathrm{AD}$ treatment on caregiver burden, premature institutionalization, and financial cost.

From the Knight Alzheimer's Disease Research Center (C.M.R., A.M.F., E.A.G., D.M.H., J.C.M.), Department of Neurology (C.M.R., A.M.F., D.M.H., J.C.M.), Hope Center for Neurological Disorders (A.M.F., D.M.H.), Departments of Pathology and Immunology (J.C.M.), Physical Therapy (J.C.M.), and Occupational Therapy (J.C.M.), and Division of Biostatistics (E.A.G.), Washington University School of Medicine, St. Louis, MO

Go to Neurology.org for full disclosures. Funding information and disclosures deemed relevant by the authors, if any, are provided at the end of the article. 
We examined associations between CSF biomarkers and incident cognitive impairment among participants followed up to 14.5 years and tested whether $\mathrm{AD}$ biomarker levels predicted future changes in function (independent activities of daily living), weight, behavior, and mood.

METHODS Standard protocol approvals, registrations, and patient consents. Study protocols were approved by the Washington University Human Research Protection Office, and written informed consent was obtained from all participants.

Participants. We analyzed annually collected data from participants enrolled in longitudinal studies at the Knight Alzheimer's Disease Research Center. Participants were recruited through word-of-mouth, advertisements, and community events for yearly assessment sessions.

Clinical assessments. The clinician's judgment about the presence of dementia was based on the principle of intraindividual change whereby the individual was used as his or her own control. Based on semistructured interviews with the participant, and separately with a collateral source who knew the participant well, the participant's cognitive and functional performance in each of 6 domains (Memory, Orientation, Judgment \& Problem Solving, Community Affairs, Home \& Hobbies, and Personal Care) was rated. These ratings were summed to yield the Clinical Dementia Rating (CDR) Sum of Boxes measure. ${ }^{5}$ An algorithm was used to calculate the global CDR. ${ }^{5} \mathrm{~A} C D R$ of 0 was taken to indicate normal cognitive functioning, whereas CDRs of $0.5,1,2$, and 3 indicated very mild, mild, moderate, and severe dementia, respectively. ${ }^{5}$ MiniMental State Examination (MMSE) ${ }^{6}$ scores were also obtained.

Weight was measured in pounds. The collateral source reported instrumental activities of daily living (IADL), which were assessed using the Functional Assessment Questionnaire (FAQ), and behavioral symptoms, using the Neuropsychiatric Inventory Questionnaire (NPI-Q). ${ }^{8}$ Depression symptoms were obtained from the participant using the Geriatric Depression Scale (GDS). ${ }^{9}$

CSF collection. CSF was obtained via lumbar puncture by trained neurologists using a 22-gauge Sprotte spinal needle to draw 20 to $30 \mathrm{~mL}$ of CSF at 8:00 AM following an overnight fast. CSF samples were gently inverted to avoid possible gradient effects and centrifuged at low speed to pellet any cellular debris and frozen at $-84^{\circ} \mathrm{C}$ after aliquoting $(0.5 \mathrm{~mL})$ into polypropylene tubes. $\mathrm{CSF}$ samples for $A \beta_{42}$, tau, and phosphorylated tau at threonine 181 $\left(\mathrm{ptau}_{181}\right)$ were analyzed using ELISA (INNOTEST; Innogenetics, Ghent, Belgium).

Inclusion criteria. Data from participants who 1) donated CSF within 1 year of a clinical assessment indicating normal cognition,

Table 1 Demographics of cognitively normal participants $(N=430)$

Age, $y$, mean (SD)

Women, $\mathrm{n}(\%)$

Minority race, $\mathrm{n}(\%)$

Education, $y$, mean (SD)

APOE $\varepsilon 4+, n(\%)$

Time between LP and baseline clinical assessment, $y$, mean (SD)

Follow-up time, $y$, mean (SD)

Abbreviation: $L P=$ lumbar puncture
2) had at least one additional clinical assessment after the baseline assessment (the assessment closest to the date of CSF donation), 3) and were aged 50 years or older at the time of the baseline assessment were included.

Statistical analyses. We tested whether the CSF biomarkers of $A \beta_{42}$, tau, $p t a u_{181}$, tau $/ A \beta_{42}$, and $p a_{181} / A \beta_{42}$ predicted each of the outcomes of interest. Mixed linear models were used to test whether baseline biomarker values predicted future decline in function (FAQ scores), weight (in pounds), behavior (NPI-Q scores), $\operatorname{mood}$ (GDS scores), and CDR Sum of Boxes and MMSE scores. Dichotomous variables reflecting normal and abnormal biomarker values were constructed using the same cutoffs used in previous research $\left(<500 \mathrm{pg} / \mathrm{mL}\right.$ for $A \beta_{42},>440 \mathrm{pg} / \mathrm{mL}$ for tau, $>78 \mathrm{pg} / \mathrm{mL}$ for $\mathrm{ptau}_{181},>0.94$ for tau $/ \mathrm{A} \beta_{42}$, and $>0.15$ for $\left.\mathrm{ptau}_{181} / \mathrm{A} \beta_{42}\right){ }^{10}$ These analyses included terms adjusting for the effects of age, sex, race (minority vs nonminority), education, and the presence of an $A P O E \varepsilon 4$ allele.

RESULTS Follow-up times ranged from 0.9 year to 14.5 years among the 430 participants meeting inclusion criteria (table 1). Thirty percent of participants (128/427) reported subjective memory or thinking concerns at baseline, answering "Yes" to the question: "Have you had any problems with your thinking or memory?" Values of each biomarker variable were associated with change across time in NPI-Q, GDS, and FAQ scores (table 2), such that abnormal levels of each biomarker were related to greater impairment with time in behavior and mood symptoms, and more difficulties with independent activities of daily living (table 2). Generally, $p$ values for the ratio variables were smaller than those associated with $A \beta_{42}$, tau, and $\mathrm{ptau}_{181}$ alone. However, biomarker levels were unrelated to weight change with time (table 2).

Abnormal biomarker values also predicted more rapidly declining performance on the MMSE and CDR Sum of Boxes compared with normal values. Fourteen participants (3.3\%) clearly developed dementia (defined as $\mathrm{CDR} \geq 1$ because some investigators consider CDR 0.5 to indicate mild cognitive impairment $[\mathrm{MCI}]^{11}$ ) over the follow-up period. We repeated the analyses after removing data from these 14 participants from the sample. Generally, results were similar, although most $p$ values reflecting the difference between the normal and abnormal biomarker groups were attenuated (table e-1 on the Neurology ${ }^{\circledR}$ Web site at www.neurology.org).

DISCUSSION Our sample showed cognitive decline with time that was linked to baseline biomarker levels. We also found that abnormal levels of CSF biomarkers predicted decline in the noncognitive outcomes of function, behavior, and mood, but were unrelated to weight changes with time.

Functionality includes the ability to perform IADL such as paying bills, shopping, and preparing meals. Decline in functional activities is an essential diagnostic criterion for $\mathrm{AD}$ dementia. Progressive 


\begin{tabular}{|c|c|c|c|}
\hline \multirow[t]{3}{*}{ Table 2} & $\begin{array}{l}\text { error) reflectin } \\
\text { al on cognitive a } \\
\text { al abnormal }\end{array}$ & $\begin{array}{l}\text { tudinal weight ch } \\
\text { cognitive outcon } \\
\text { iomarker values }\end{array}$ & \multirow[t]{3}{*}{$\begin{array}{l}\text { and } \\
\text { a } \\
\text { eline }\end{array}$} \\
\hline & \multicolumn{2}{|c|}{ Baseline biomarker value } & \\
\hline & Normal & Abnormal & \\
\hline \multicolumn{4}{|l|}{$A \beta_{42}$} \\
\hline No. & 272 & 158 & \\
\hline Weight, lb & $-0.769(0.862)$ & $-0.059(1.068)$ & 0.613 \\
\hline FAQ & $0.084(0.046)$ & $0.296(0.062)$ & 0.006 \\
\hline GDS & $0.022(0.021)$ & $0.124(0.028)$ & 0.004 \\
\hline NPI-Q & $0.016(0.032)$ & $0.127(0.041)$ & 0.035 \\
\hline MMSE & $-0.013(0.019)$ & $-0.154(0.025)$ & $<0.001$ \\
\hline CDR Sum of Boxes & $0.041(0.016)$ & $0.159(0.022)$ & $<0.001$ \\
\hline \multicolumn{4}{|l|}{ tau } \\
\hline No. & 358 & 72 & \\
\hline Weight, lb & $0.114(0.776)$ & $-2.271(1.33)$ & 0.115 \\
\hline FAQ & $0.106(0.040)$ & $0.422(0.090)$ & 0.001 \\
\hline GDS & $0.035(0.019)$ & $0.171(0.039)$ & 0.002 \\
\hline NPI-Q & $0.025(0.028)$ & $0.190(0.057)$ & 0.010 \\
\hline MMSE & $-0.047(0.018)$ & $-0.131(0.037)$ & 0.041 \\
\hline CDR Sum of Boxes & $0.062(0.015)$ & $0.191(0.033)$ & $<0.001$ \\
\hline \multicolumn{4}{|l|}{ ptau $_{181}$} \\
\hline No. & 355 & 75 & \\
\hline Weight, lb & $0.082(0.773)$ & $-2.190(1.317)$ & 0.130 \\
\hline FAQ & $0.116(0.040)$ & $0.366(0.090)$ & 0.012 \\
\hline GDS & $0.039(0.019)$ & $0.152(0.039)$ & 0.012 \\
\hline NPI-Q & $0.023(0.028)$ & $0.199(0.057)$ & 0.006 \\
\hline MMSE & $-0.042(0.017)$ & $-0.156(0.037)$ & 0.006 \\
\hline CDR Sum of Boxes & $0.062(0.015)$ & $0.188(0.033)$ & $<0.001$ \\
\hline \multicolumn{4}{|l|}{$\operatorname{tau} / \mathbf{A} \beta_{42}$} \\
\hline No. & 360 & 70 & \\
\hline Weight, lb & $-0.788(0.732)$ & $1.037(1.673)$ & 0.322 \\
\hline FAQ & $0.108(0.040)$ & $0.439(0.093)$ & 0.001 \\
\hline GDS & $0.029(0.018)$ & $0.233(0.042)$ & $<0.001$ \\
\hline NPI-Q & $0.021(0.027)$ & $0.268(0.061)$ & $<0.001$ \\
\hline MMSE & $-0.032(0.017)$ & $-0.236(0.038)$ & $<0.001$ \\
\hline CDR Sum of Boxes & $0.053(0.014)$ & $0.247(0.033)$ & $<0.001$ \\
\hline \multicolumn{4}{|l|}{$\operatorname{ptau}_{181} / A \beta_{42}$} \\
\hline No. & 343 & 87 & \\
\hline Weight, lb & $-0.667(0.778)$ & $-0.116(1.318)$ & 0.726 \\
\hline FAQ & $0.105(0.041)$ & $0.386(0.083)$ & 0.003 \\
\hline GDS & $0.023(0.019)$ & $0.202(0.036)$ & $<0.001$ \\
\hline NPI-Q & $0.010(0.028)$ & $0.238(0.053)$ & $<0.001$ \\
\hline MMSE & $-0.024(0.017)$ & -0.217 (0.033) & $<0.001$ \\
\hline CDR Sum of Boxes & $0.050(0.015)$ & 0.219 (0.029) & $<0.001$ \\
\hline
\end{tabular}

Abbreviations: $A \beta_{42}=\beta$-amyloid 42; CDR = Clinical Dementia Rating; FAQ = Functional Assessment Questionnaire; GDS = Geriatric Depression Scale; MMSE = Mini-Mental State Examination; NPI-Q = Neuropsychiatric Inventory Questionnaire; $\operatorname{ptau}_{181}=$ phosphorylated tau at threonine 181. functional decline leads to dependence, which decreases the patient's quality of life, places physical and psychological burdens on the caregiver, and can lead to institutionalization. A previous cross-sectional study found that amyloid burden, imaged using Pittsburgh compound B, correlated with worse performance on IADL among persons with $\mathrm{MCI},{ }^{12}$ but there was no cross-sectional relationship between Pittsburgh compound B uptake and IADL among 19 individuals with normal cognition. ${ }^{12}$ However, a longitudinal study found that CSF biomarkers of $A \beta_{42}$, tau, and ptau $_{181}$ predicted future decline on IADL among participants who were cognitively nor$\mathrm{mal}$ at baseline, ${ }^{13}$ consistent with our results.

An earlier study found that among participants with $A D$, the CSF biomarker $A \beta_{42}$ was correlated with the presence of aggressive behaviors. ${ }^{14}$ Aggression was not linked to levels of CSF tau or ptau ${ }_{181}$, and none of the biomarkers were correlated with any of the other behaviors studied, including delusions, hallucinations, anxiety, and agitation. ${ }^{14}$ Cross-sectional studies show conflicting results regarding mood, with reports that CSF $A \beta_{42}$ is increased, ${ }^{15}$ decreased, ${ }^{16}$ and unrelated ${ }^{14}$ to depression. However, depression has been found to be associated with greater numbers of amyloid plaques ${ }^{17,18}$ and neurofibrillary tangles at autopsy. ${ }^{17,19}$ To our knowledge, no previous studies have investigated whether abnormal biomarkers in cognitively normal adults predict future behavioral symptoms. We found that depression scores and other behavioral symptoms monitored by the NPI-Q worsen faster among individuals with preclinical $\mathrm{AD}$ at baseline as defined by CSF biomarkers.

Weight loss is associated with cognitive impairment, with $\mathrm{AD}$ onset, and increases the risk of death. A cross-sectional study found that abnormal CSF levels of $A \beta_{42}$ and tau were associated with lower body mass index in a sample comprising individuals with and without memory problems (i.e., were cognitively normal, had MCI, or had AD dementia), ${ }^{20}$ consistent with a possible link between abnormal $\mathrm{AD}$ biomarker levels and weight loss. A literature review did not reveal any prior studies that examined biomarkers as a predictor of future weight loss among cognitively normal individuals. Our nonsignificant results suggest that if such a link exists, the relationship is weaker than the association between CSF biomarkers and the other noncognitive outcomes studied here.

A limitation of the study is that we conducted several statistical tests, and it is possible that some statistical tests were significant by chance. The likelihood

Slope estimates were adjusted for the effects of age, sex, race, education, and the presence of an APOE $\varepsilon 4$ allele.

a Lower scores on the MMSE indicate greater impairment. Higher scores on the other tests indicate greater impairment. 
of a statistical test being significant by chance decreases with smaller $p$ values.

We found that CSF biomarkers among cognitively normal individuals are associated with future decline in some, but not all, noncognitive $\mathrm{AD}$ symptoms studied. Future research should test whether the ratios of tau $/ A \beta_{42}$ and $\mathrm{ptau}_{181} / \mathrm{A} \beta_{42}$ are better predictors of decline in noncognitive outcomes compared with the individual molecular markers alone.

\section{AUTHOR CONTRIBUTIONS}

Dr. Roe: study concept and design, data analysis and interpretation, drafting and critical revision of manuscript. Dr. Fagan: data acquisition, analysis and interpretation, critical revision of manuscript, study supervision. Dr. Grant: data acquisition, analysis and interpretation, critical revision of manuscript. Dr. Holtzman: data analysis and interpretation, critical revision of manuscript. Dr. Morris: data acquisition, analysis and interpretation, critical revision of manuscript, study supervision.

\section{ACKNOWLEDGMENT}

The authors thank the participants, investigators, and staff of the Knight Alzheimer's Disease Research Center Clinical (participant assessments) and Genetics Cores (genotyping), and the investigators and staff of the Biomarker Core (P01 AG026276) for CSF analytes.

\section{STUDY FUNDING}

Supported by the Longer Life Foundation, the National Institute of Neurological Disorders and Stroke (P30 NS057105), National Institute on Aging (P50 AG005681, P01 AG003991, and P01 AG026276), Fred Simmons and Olga Mohan, the Farrell Family Research Fund, and the Charles and Joanne Knight Alzheimer's Research Initiative of the Washington University Knight Alzheimer's Disease Research Center

\section{DISCLOSURE}

C. Roe reports no disclosures. A. Fagan is supported by grants from the National Institute on Aging of the NIH (P01 AG03991, P01 AG026276, and U01 AG032438) and the Hope Center for Neurological Disorders, and is a member of the Alzheimer's Disease CSF Biomarker Development Advisory Board for Roche and the US Alzheimer's Disease Advisory Board for Lilly USA. No conflict of interest exists. E. Grant reports no disclosures. D. Holtzman receives research grants to his laboratory from Eli Lilly, AstraZeneca, and Pfizer. He is on the scientific advisory board of Pfizer and C2N Diagnostics. He has consulted for Bristol-Myers Squibb. He is a cofounder of C2N Diagnostics. J. Morris reports disclosures: neither Dr. Morris nor his family owns stock or has equity interest (outside of mutual funds or other externally directed accounts) in any pharmaceutical or biotechnology company. Dr. Morris has participated or is currently participating in clinical trials of antidementia drugs sponsored by Janssen Immunotherapy and Pfizer. Dr. Morris has served as a consultant for the following companies: Eisai, Esteve, Janssen Alzheimer Immunotherapy Program, GlaxoSmithKline, Novartis, and Pfizer. He receives research support from Eli Lilly/Avid Radiopharmaceuticals and is funded by NIH grants P50AG005681, P01AG003991, P01AG026276, and U19AG032438. Go to Neurology.org for full disclosures.

Received June 3, 2013. Accepted in final form September 5, 2013.

\section{REFERENCES}

1. Fagan AM, Head D, Shah AR, et al. Decreased cerebrospinal fluid $A \beta 42$ correlates with brain atrophy in cognitively normal elderly. Ann Neurol 2009;65:176-183.

2. Jack CR Jr, Knopman DS, Jagust WJ, et al. Hypothetical model of dynamic biomarkers of the Alzheimer's pathological cascade. Lancet Neurol 2010;9:119-128.

3. Sperling RA, Aisen PS, Beckett LA, et al. Toward defining the preclinical stages of Alzheimer's disease: recommendations from the National Institute on Aging-Alzheimer's Association workgroups on diagnostic guidelines for Alzheimer's disease. Alzheimers Dement 2011;7:280-292.

4. Zekry D, Graf CE, Giannelli SV, Gold G, Michel JP. Noncognitive outcomes in trials of disease-modifying drugs for Alzheimer's disease. Eur Geriatr Med 2012;3:37-42.

5. Morris JC. The Clinical Dementia Rating (CDR): current version and scoring rules. Neurology 1993;43:2412-2414.

6. Folstein MF, Folstein SE, McHugh PR. "Mini-Mental State": a practical method for grading the cognitive state of patients for the clinician. J Psychiatr Res 1975;12:189-198.

7. Pfeffer RI, Kurosaki TT, Harrah CH Jr. Measurement of functional activities in older adults in the community. J Gerontol 1982;37:323-329.

8. Kaufer DI, Cummings JL, Ketchel P, et al. Validation of the NPI-Q, a brief clinical form of the Neuropsychiatric Inventory. J Neuropsychiatry Clin Neurosci 2000;12: 233-239.

9. Sheikh JI, Yesavage JA. Geriatric Depression Scale (GDS): recent evidence and development of a shorter version. In: Brink TL, editor. Clinical Gerontology: A Guide to Assessment and Intervention. New York: The Haworth Press; 1986:165-173.

10. Tarawneh R, D'Angelo G, Macy E, et al. Visinin-like protein-1: diagnostic and prognostic biomarker in Alzheimer disease. Ann Neurol 2011;70:274-285.

11. Petersen RC, Doody R, Kurz A, et al. Current concepts in mild cognitive impairment. Arch Neurol 2001;58: 1985-1992.

12. Marshall GA, Olson LE, Frey MT, et al. Instrumental activities of daily living impairment is associated with increased amyloid burden. Dement Geriatr Cogn Disord 2011;31:443-450.

13. Okonkwo OC, Alosco ML, Griffith HR, et al. Cerebrospinal fluid abnormalities and rate of decline in everyday function across the dementia spectrum: normal aging, mild cognitive impairment, and Alzheimer disease. Arch Neurol 2010;67:688-696.

14. Engelborghs S, Maertens K, Vloeberghs E, et al. Neuropsychological and behavioural correlates of CSF biomarkers in dementia. Neurochem Int 2006;48:286-295.

15. Gudmundsson P, Skoog I, Waern M, et al. The relationship between cerebrospinal fluid biomarkers and depression in elderly women. Am J Geriatr Psychiatry 2007;15:832-838.

16. Pomara N, Bruno D, Sarreal AS, et al. Lower CSF amyloid beta peptides and higher F2-isoprostanes in cognitively intact elderly individuals with major depressive disorder. Am J Psychiatry 2012;169:523-530.

17. Rapp MA, Schnaider-Beeri M, Grossman HT, et al. Increased hippocampal plaques and tangles in patients with Alzheimer disease with a lifetime history of major depression. Arch Gen Psychiatry 2006;63:161-167.

18. Meynen G, Van Stralen H, Smit JH, Kamphorst W, Swaab DF, Hoogendijk WJG. Relation between neuritic plaques and depressive state in Alzheimer's disease. Acta Neuropsychiatrica 2010;22:14-20.

19. Rapp MA, Schnaider-Beeri M, Purohit DP, Perl DP, Haroutunian V, Sano M. Increased neurofibrillary tangles in patients with Alzheimer disease with comorbid depression. Am J Geriatr Psychiatry 2008;16:168-174.

20. Ewers M, Schmitz S, Hansson O, et al. Body mass index is associated with biological CSF markers of core brain pathology of Alzheimer's disease. Neurobiol Aging 2012; 33:1599-1608. 